\title{
Ethical spaces in imperfect global health collaborations. A commentary on Keynejad's research, education and capacity building initiative to address gender-based violence in the Global South
}

\author{
Hanna Kienzler ${ }^{1}$ (D) \\ Received: 12 May 2021 / Accepted: 5 December 2021 \\ (c) The Author(s), under exclusive licence to Springer-Verlag GmbH Germany 2021
}

Violence is a social phenomenon. It carries meaning, manifests itself in material and non-material forms, and has communicative effects, symbolic use, and performative quality [1]. Violence is capable of transmitting messages that devalue or destroy previously held referents, social conventions, and identities [2]. Gender-Based Violence (GBV) is one way in which violence manifests itself in the everyday. It is considered a clear marker of deep-rooted gender inequality and discrimination, with women everywhere facing high risks of sexual abuse and Intimate Partner Violence in their lifetime [3]. It is recognised to be an important determinant of health, and various United Nation bodies are calling to prioritise its prevention, and to address its physical and mental health impacts. Yet, there is limited research on the intersection of GBV and mental health and, related to this, prevention and treatment. Moreover, there is scarce information on education and capacity strengthening priorities in the area of violence, abuse, and mental health globally.

The study by Roxanne Keynejad and colleagues focuses on the understudied field GBV and its impact on mental health in the context of the Global South. They conducted a qualitative survey to explore, from the perspectives of academics and voluntary sector workers, the underlying reasons for prominent knowledge, and action gaps in GBV and

This article belongs to the Topical Collection Violence and Women's Mental Health.

This commentary refers to the article available at https://doi.org/ 10.1007/s00127-021-02061-5.

Hanna Kienzler

hanna.kienzler@kcl.ac.uk

1 Department of Global Health \& Social Medicine, King's College London, Bush House (NE) 3.15, 40 Aldwych, London WC2B 4BG, UK mental health; priorities for research, education, and capacity strengthening; and recommendations for action. The survey findings highlight clearly that participants residing and working in the Global South face fundamental shortcomings in GBV and mental health education and training, access to funding, influence in terms of research conceptualisation and design, and knowledge generation and dissemination opportunities.

Survey participants pointed to key barriers and avenues for change in GBV and mental health research in countries of the Global South on various levels. On practical and operational levels, they identified lack of funding, limited support for sustainable programme development, high workforce turnover, and arduous ethical approval processes. On ideological and leadership levels, they highlighted cultural sensitivities and stigma, normalisation of GBV where it was merely seen as reflective of unquestioned patriarchal societal norms, and the lack of political will where leaders prefer to overlook GBV and, consequently, fail to include it in their programming.

To address such intricate challenges, survey participants demanded open collaborative structures capable of bringing experts from the Global South and Global North together to strengthen research capacity, offer academic and practical training in trauma-informed approaches to GBV and mental health, and co-produce interventions with GBV survivors. Furthermore, they suggested the development of "incubation hubs" for new knowledge development, training, and intervention to take place on a global scale. A cohort of "champions" was envisioned to build links with existing networks (e.g., the African Alliance for Maternal Mental Health or the International Marce Society for Perinatal Mental Health) and regional and national decision-makers "to facilitate research and overcome administrative and ideological barriers." 
In response to the survey findings, Keynejad and team propose the development of interdisciplinary networks as one way to achieve more equitable collaboration and generate contextual understandings of the intersection of GBV and mental illness, capacity strengthening opportunities, and feasible evidence-based interventions responsive to the needs of GBV survivors in countries of the Global South. The authors emphasise that such networks would need to be built on trusting working relationships over the long term to have the potential to overcome cultural barriers to addressing sensitive concerns such as gender inequality, GBV, and mental health in different locations.

The creation of such international networks and "incubation hubs" based on multi-country and interdisciplinary collaboration could indeed have the potential to build vital partnerships between Global South and Global North scholars and practitioners, and to foster locally driven development and programmes to address GBV and mental health. I would like to caution though that it will not be enough to bring diverse communities of scholars and practitioners into the same space without breaking through some of the dominant patters of Global South-Global North collaboration. Such patterns are characterised by structural inequalities and discrepancies in power which often lead to Global South researchers and practitioners being on the implementation side of research (e.g., data collection), while their Global North counter parts conceptualise and design the purpose, priority, and outcome of research and intervention $[4,5]$.

Destabilising such patterns is not straightforward in a context where Global North researchers and practitioners tend to actively "unknow" or even avoid straightforward talk about inequalities between themselves and their Global South colleagues [6]. Conversely, Global South researchers and practitioners are often afraid to rock the boat concerned that their critique might lead to unrepairable conflicts with their high-income country colleagues who hold the strings to funding, equipment and other scientific resources, and strategic networks. Such dynamics have been shown to be harmful for shared goal development, creation of more equal relationships, and improvement of research quality [6].

The feminist writer McIntosh [7] reminds us that "to redesign social systems we need first to acknowledge their colossal unseen dimensions." One of these unseen dimensions in global health and development work is the legacy of colonialism which manifests itself in various forms until today. For instance, Global North institutions, decisionmakers, practitioners, and scholars largely determine what knowledge counts as they control resources and budgets, dominate the publishing industry, and lead on capacity building and training programmes. This arrangement has been shown to lead to the establishment and maintenance of an institutionalised monoculture whereby Western-European modes of thinking, knowing, and acting are deemed singular and universal $[8,9]$. At the same time, realities, knowledges, and experiences of experts from other sociocultural backgrounds are neglected or actively silenced, social injustices are widened, and the value of different histories and epistemologies for creating a diverse global knowledge is overlooked [10]. Such entrenched power inequalities beg the questions what it would take to destabilise hegemonic practices and how we can begin to create spaces for a plurality of knowledge, experiences, and histories to co-exist on equal footing.

Linking these questions back to Keynejad and colleagues' ambition to create international networks to address GBV and mental health in the Global South, I would like to encourage a shift in attention to network internal power inequalities and the ways in which these shape and impact on collaborative working. There is no one way to do this. Personally, I found the engagement with Willie Ermine's concept of the "ethical space" inspirational [8]. ${ }^{1}$ The ethical space is considered to be an invisible but energy laden field that is created when societies or groups with disparate worldviews come together to engage in cross-cultural conversations in the pursuit of ethically practicing diversity. That is, it recognises difference and diversity between these communities, because each "entity is moulded from a distinct history, knowledge tradition, philosophy, and social and political reality." Furthermore, each brings their own ethical standards of the good, responsibility, and obligation, yet needs to recognise that these same standards might infringe or violate the space of others.

Therefore, how can we co-exist as equals in imperfect collaborative arrangements? From Ermine, we can learn that "the notion of an agreement to interact must always be preceded by the affirmation of human diversity created by philosophical and cultural difference." This, he recognises may be daunting for all involved as it involves dialogical engagements that address issues as complex as language, distinct histories, knowledge traditions, values, interests, and social, economic and political realities, and how these shape and impact an agreement to interact.

The proposal might also be uncomfortable for those working in privileged conditions, because it requires Global North scholars and practitioners to dismantle their own power. It is no longer good enough to talk about 'partnerships' and 'collaborations' when failing to address unequal opportunities, salaries, and working and living conditions in the Global South $[4,6]$. It further requires a recognition that knowledge and skills exchanges are two-way-streets where differently positioned actors are willing to learn from each other. Too often, researchers and practitioners from

\footnotetext{
${ }^{1}$ I would like to thank one of the reviewers for pointing me to Ermine's important work.
} 
countries in the Global North dismiss the expertise and know-how developed by their Global South counterparts and, thereby, fail to strengthen their own capacity when it comes to improving cultural competencies, research design and methods, pedagogical capacity, and access opportunities for mentorship [11].

The survey results by Keynejad and colleagues make evident that GBV and mental health research in the Global South would benefit from addressing power and resource asymmetries between Global North and Global South partners. I would like to push their thinking and practice even further by encouraging them to actively create ethical spaces so as to engage in substantive, reflexive, and deep dialogue with their colleagues from different social and cultural communities. I believe that it is only thereby that we can ensure that diverse knowledges, epistemologies, and languages can influence and impact on global health. The proposed networks could thus be fertile grounds whereas colleagues and I have argued previously, "a real transformation would see researchers and educators [from the Global South] confidently engage with, and use, local methods and paradigms in the international realm as they design projects, collect data and develop theory" [10].

Funding There are no funding sources.

Availability of data and materials Not applicable.

Code availability Not applicable.

\section{Declarations}

Conflict of interest The author declares that there is no conflict of interest.

Ethical approval Not applicable.

\section{References}

1. Kienzler H, Locke P (2017) The effects of societal violence in war and post-war contexts. In: White R, Orr D, Jain S, Read U (eds) The Palgrave handbook of global mental health: socio-cultural perspectives. Palgrave Macmillan, UK, pp 285-305

2. Das V, Kleinman A (2000) Introduction. In: Das V (ed) Violence and subjectivity. University of California Press, Berkeley, pp 1-18

3. Zamora E, Burgess R, Fonseca L, González-Gort M, Kakuma R (2021) Gender, mental health and resilience in armed conflict: listening to life stories of internally displaced women in Colombia. https://doi.org/10.31235/osf.io/4kar5

4. Boum Y II, Burns BF, Siedner M, Mburu BE, Haberer JE (2018) Advancing equitable global health research partnerships in Africa. BMJ Glob Health. https://doi.org/10.1136/bmjgh-2018-000868

5. Minn P (2015) Troubling objectivity: the promises and pitfalls of training Haitian clinicians in qualitative research methods. Med Anthropol 34(1):39-53. https://doi.org/10.1080/01459740.2014. 961064

6. Geissler PW, Okwaro F (2014) Developing world: discuss inequality. Nature News 513(7518):303

7. McIntosh P (1998) White privilege: unpacking the invisible Knapsack. Peace and Freedom https://psychology.umbc.edu/files/2016/ 10/White-Privilege_McIntosh-1989.pdf

8. Ermine W (2007) The ethical space of engagement. Indigenous Law J 6(1):193-204

9. Abimbola $S$ (2019) The foreign gaze: authorship in academic global health. BMJ Glob Health 4(5):1-5. https://doi.org/10.1136/ bmjgh-2019-002068

10. Tamimi N, Kienzler H, Hammoude W, Giacaman R (in press) Toward decolonising research methods training: The development of a locally responsive online learning course on research methods for mental health in war and conflict for researchers and practitioners in the Gaza Strip. Global Mental Health.

11. Murphy J, Hatfield J, Afsana K, Neufeld V (2015) Making a commitment to ethics in global health research partnerships: a practical tool to support ethical practice. J Bioethical Inquiry 12(1):137-146. https://doi.org/10.1007/s11673-014-9604-6 